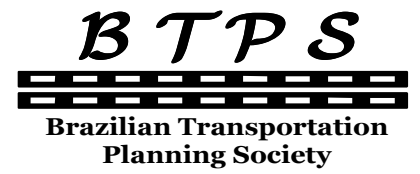

Journal of Transport Literature

Vol. 7, n. 3, pp. 240-269, Jul. 2013

JTL|RELIT

Research Directory www.transport-literature.org
ISSN 2238-1031

\title{
Governança em plataformas logísticas: uma análise dos elementos e atributos a serem considerados neste tipo de empreendimento logístico
}

[Governance in logistics platforms: an analysis of the elements and attributes to be considered in this type of logistic enterprise]

\author{
Rafael Mozart da Silva*, Eliana Terezinha Pereira Senna, \\ Luiz Afonso dos Santos Senna, Orlando Fontes Lima Júnior
}

Universidade Estadual de Campinas - UNICAMP - Brasil, Universidade Estadual de Campinas - UNICAMP - Brasil, Universidade Federal do Rio Grande do Sul - UFRGS - Brasil, Universidade Estadual de Campinas - UNICAMP - Brasil

Submitted 12 Dec 2012; received in revised form 11 Jan 2013; accepted 13 Jan 2013

\begin{abstract}
Resumo
As plataformas logísticas são empreendimentos logísticos de grande porte que atendem a uma área ou região, onde se concentram e realizam-se atividades voltadas a produção, transportes, logística e distribuição de mercadorias, utilizando-se de uma mesma base de serviços onde os diferentes atores envolvidos se relacionam com objetivo de obter uma maior eficiência em suas operações. Esta pesquisa teve como objetivo identificar os atributos e elementos que podem ser aplicados à governança das plataformas logísticas. Para atingir o objetivo deste trabalho, foi realizado uma revisão bibliográfica sobre os principais conceitos relacionados a governança e plataformas logísticas e também uma pesquisa das características das plataformas logísticas Europeias, extraídas dos relatórios Feasibility of Freight Villages in the NYMTC Region e An Exploration of the Freight Village Concept and its Applicability to Ontario. Como resultado verificou-se que a governança esta associada influência do poder, o grau de hierarquia entre os diferentes atores envolvidos ou participantes e também aos níveis e sinergias que se estabelecem nas relações interorganizacionais tanto na esfera pública como privada.
\end{abstract}

Palavras-Chave: plataforma logística, governança logística, empreendimento logístico, infraestrutura logística.

\begin{abstract}
The Logistics Platforms are logistics enterprises of large scale that serve an area or region,where they concentrate and realized activities directed to production, transport, logistics and goods distribution, using the same basis of services where different actors involved are related to goal of achieving greater efficiency in their operations. This research aimed to identify the attributes and elements that can be applied to the governance of logistics platforms. To reach the purpose of this research, it was realized a literature review about the main concepts related to governance and logistics platforms and also a survey of features of logistics platforms located in Europe extracted from reports Feasibility of Freight Villages in the NYMTC Region and An Exploration of the Freight Village Concept and Its Applicability to Ontario. As a result it was found that the governance of this type of enterprise is associated influence of power, the degree of hierarchy between the different actors involved or participating and also the levels and synergies that are established in interorganizational relationships both in the public and private spheres.
\end{abstract}

Key words: logistics platform, logistics governance, enterprise logistics, logistics infrastructure.

* Email: rafmozart@terra.com.br.

\section{Recommended Citation}

Silva, R. M. , Senna, E. T. P., Senna, L. A. D.S. and Lima Júnior, O. F. (2013) Governança em plataformas logísticas: uma análise dos elementos e atributos a serem considerados neste tipo de empreendimento logístico. Journal of Transport Literature, vol. 7, n. 3, pp. 240-269.

- JTL/RELIT is a fully electronic, peer-reviewed, open access, international journal focused on emerging transport markets and published by BPTS - Brazilian Transport Planning Society. Website www.transport-literature.org. ISSN 2238-1031.

This paper is downloadable at www.transport-literature.org/open-access. 


\section{Introdução}

A utilização de um sistema de operações logísticas que possa integrar as atividades e também os atores envolvidos na cadeia de abastecimento torna-se cada vez mais uma preocupação estratégica para as organizações. A busca por um adequado nível de serviço junto ao cliente e também a busca por uma manutenção eficiente dos custos tem levado as organizações a repensarem suas estratégias logísticas, resultando em muitos casos na centralização da produção e da atividade de distribuição baseada no tempo (Cambra-Fierro e Ruiz-Benitez, 2009; Trappey et al. 2011).

A gestão da cadeia de suprimentos tem o papel de coordenação estratégica e sistêmica das tradicionais funções de negócios e das táticas ao longo dessas funções de negócios no âmbito de uma determinada empresa e ao longo dos negócios no âmbito da cadeia de suprimentos, com objetivo de aperfeiçoar o desempenho a longo prazo das empresas isoladamente e da cadeia de suprimentos como um todo (Mentzer et al. 2001; Matera, 2012).

A fim de aproximar alguns dos objetivos da cadeia de suprimentos, que é a manutenção do nível de serviço e também os baixos custos globais ao longo da rede, existe a necessidade da criação de meios específicos de coordenação para gerenciar as intenções e interações entre os parceiros. As plataformas logísticas tem um importante papel de facilitação para o fornecimento de um serviço complexo para a comunicação e avaliação das informações relevantes que possam impactar as operações dos canais de abastecimento (Váncza et al. 2010).

O objetivo deste trabalho foi identificar os atributos e elementos que podem ser aplicados à governança das plataformas logísticas. Para atingir o objetivo desta pesquisa, realizou-se; i) revisão bibliográfica sobre os principais conceitos aplicáveis a governança de plataformas logísticas e, ii) levantamento das características das plataformas logísticas localizadas na Europa, extraídas dos relatórios Feasibility of Freight Villages in the NYMTC Region e An Exploration of the Freight Village Concept and its Applicability to Ontario (Boile et al. 2009; Higgins e Ferguson, 2011). 
A plataforma logística é um local específico onde as atividades relativas à produção, transportes, logística e distribuição de mercadorias são realizadas, utilizando-se de uma mesma base de serviços onde os diferentes atores envolvidos se relacionam com objetivo de obter uma maior eficiência em suas operações (Rimienė e Grundey, 2007; Meidutė, 2007; Pettit e Beresford, 2009).

Neste contexto percebe-se a importância de se verificar a forma de governança das plataformas logísticas por serem considerados arranjos logísticos complexos. Os arranjos logísticos compreendem sistemas complexos sendo influenciado por diferentes variáveis, dentre as quais se destaca a sua forma de governança que pode impactar o desempenho das partes envolvidas neste arranjo (Cooper e Ellram, 1993; Pfohl e Buse, 2000).

Na Seção 1, apresenta-se a metodologia e as etapas de pesquisa utilizadas neste trabalho. O referencial teórico relacionado a plataformas logísticas e os principais aspectos relacionados à governança são apresentados na Seção 2. Uma síntese das características das plataformas logísticas na Europa extraídas dos relatórios é apresentada na Seção 3. Na Seção 4, são discutidos a aplicabilidade dos elementos de governança da cadeia de suprimentos à governança em plataformas logísticas. Por fim, demonstram-se as conclusões e contribuições do trabalho, as quais poderão ser utilizadas como hipóteses para novas pesquisas.

\section{Metodologia de Pesquisa}

Buscando identificar as principais características voltadas a governança das plataformas logísticas, o enfoque desta pesquisa é qualitativo. De acordo com Creswell (2007), as pesquisas em que as variáveis ainda são desconhecidas, a análise qualitativa mostra-se a mais adequada. Em uma abordagem qualitativa, a quantidade de informações é substituída pela intensidade, mediante a análise de diferentes fontes que possam ser relacionadas, atingindo, desta forma, níveis de compreensão que não podem ser alcançados por meio de uma pesquisa quantitativa.

$\mathrm{Na}$ construção do referencial teórico que serviu de base para o desenvolvimento deste trabalho, utilizou-se a pesquisa bibliográfica que é o estudo sistematizado desenvolvido com base no material já publicado, através de livros, revistas, relatórios, jornais, artigos, meios 
eletrônicos dentre outros, isto é material acessível ao publico em geral (Marconi e Lakatos, 2009). A Figura 1 ilustra as principais etapas para o desenvolvimento desta pesquisa.

\section{Figura 1 - Etapas do método de trabalho ${ }^{1}$}

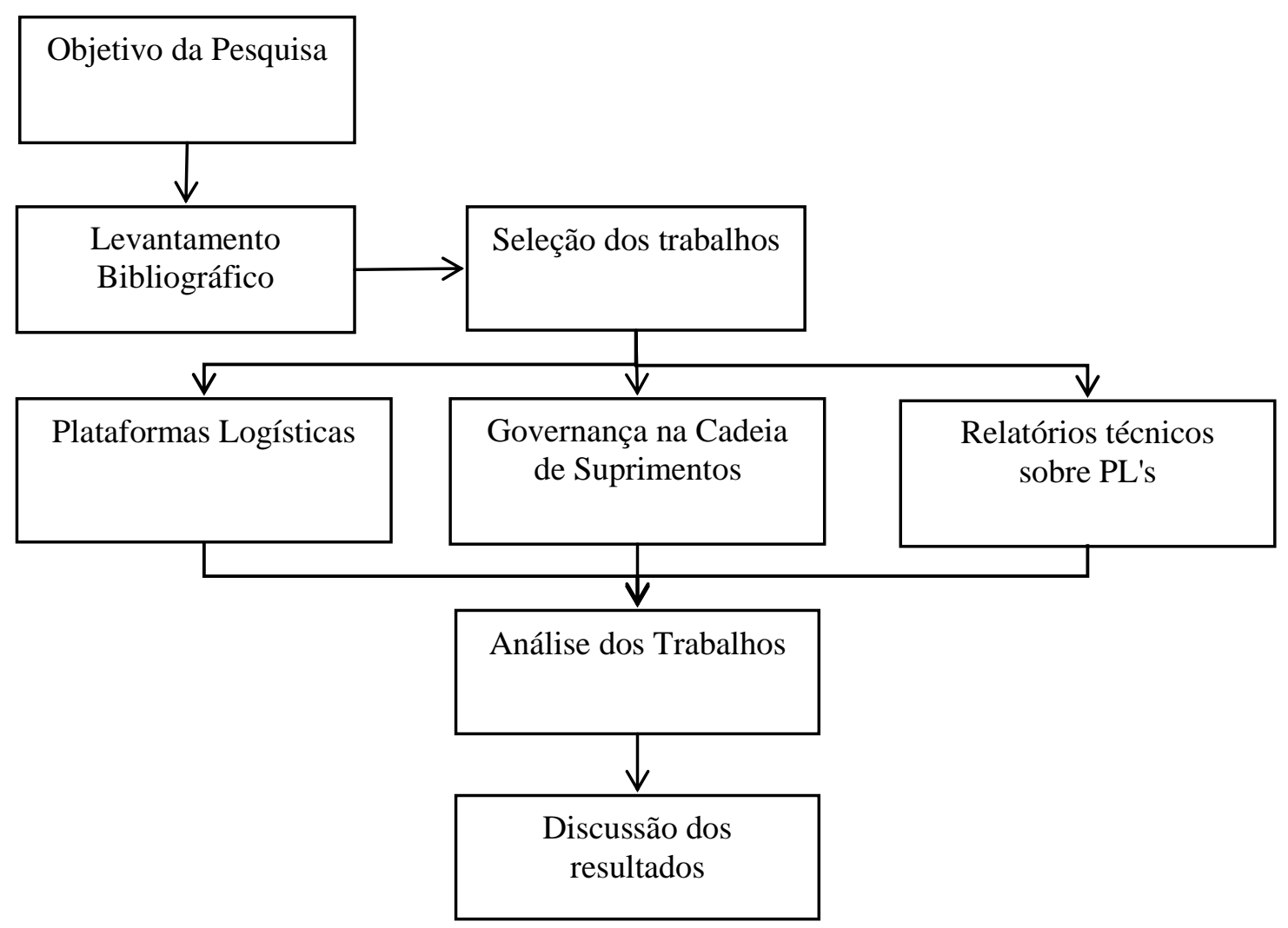

Para a realização do levantamento bibliográfico sobre o tema de governança em plataformas logísticas e também na cadeia de suprimentos, foram realizadas pesquisas na base de dados da Coordenação de Aperfeiçoamento de Pessoal de Nível Superior - CAPES de trabalhos acadêmicos como artigos, dissertações de mestrado, teses de doutorado dentre outros documentos. A partir do referencial teórico foi possível identificar os conceitos sobre plataformas logísticas e as características de governança da cadeia de suprimentos que têm aderência à governança de plataformas logísticas.

A fim de evidenciar as características de plataformas logísticas existentes atualmente, foram analisados os relatórios técnicos Feasibility of Freight Villages in the NYMTC Region e An Exploration of the Freight Village Concept and its Applicability to Ontario (Boile et al. 2009; Higgins e Ferguson, 2011).

\footnotetext{
${ }^{1}$ Fonte: Elaborada pelos autores.
} 


\section{Referencial Teórico}

Nesta seção apresenta-se os conceitos e características relevantes para a compreensão sobre as plataformas logísticas e também a fundamentação teórica relativa a governança na cadeia de suprimentos, visando dar sustentação teórica ao objetivo do estudo proposto.

\subsection{Plataforma Logística - PL}

Percebe-se atualmente a necessidade de uma reorganização dos arranjos logísticos, os quais possam atender as funções de abastecimento de materiais e também cobrir a extensão geográfica da cadeia suprimentos e as alterações na organização do território, pois o desenvolvimento organizado das atividades logísticas possibilita um nível de serviço adequado aos clientes, assim como uma redução dos custos e também tornam ágeis a circulação de mercadorias e o fluxo de informações. Neste contexto as plataformas logísticas - PL's buscam reunir de forma eficiente e racionalizada os diversos tipos de recursos ao menor custo possível e níveis de serviço adequados aos diferentes elos da cadeia logística (Duarte, 2004; Rosa, 2005; Kobayashi, 2000; Dubke et al. 2004).

O conceito de plataforma logística (PL) foi desenvolvido na França pelo Groupement Européam D'intérêt Economique (GEIE) com o objetivo de substituir os ineficientes serviços prestados pelos terminais de cargas. A plataforma logística é uma zona delimitada onde são realizadas por diversos operadores logísticos, as atividades relativas a logística regional, nacional e internacional. A localização da PL esta relacionada com a ligação entre os diferentes tipos de modais, sendo que a sua implementação tem como base os estudos sobre os fluxos de insumos, mercadorias e serviços, associados a uma avaliação de seus nós de suprimento e distribuição, racionalizando a relação custo \& benefício (Boudouin, 1996; Colin, 1996).

Para European Association of Freight Village - Europlataforms uma associação de plataformas logísticas na Europa define estes empreendimentos logísticos como uma zona delimitada, no interior da qual se exerçam, por diferentes operadores, todas as atividades relativas ao transporte, a logística e a distribuição de mercadorias, tanto para o trânsito nacional como para o internacional. Estes operadores podem ser proprietários, arrendatários dos edifícios, equipamentos, instalações (armazéns, áreas de estocagem, oficinas) que estão 
construídos e operam dentro do condomínio do empreendimento. Uma plataforma deve ter um regime de livre concorrência para todas as empresas interessadas pelas atividades acima descritas e compreender serviços comuns para as pessoas e para os veículos dos usuários. A gestão destas zonas logísticas deve ser realizada por uma única entidade, seja ela pública, privada ou mista (Europlatforms, 2004).

Outro conceito de plataforma logística é abordado pelo Telecotrans - Plataformas Logísticas e Centros de Transportes de Mercadorias na Espanha (1999), no qual apresenta as PL's como pontos ou áreas de rupturas das cadeias de transporte e logística, nos quais concentram atividades e funções técnicas e de valor agregado. As plataformas logísticas consistem em instalações ou áreas capazes de agregar valor na cadeia de suprimentos ou de distribuição através das operações logísticas que são realizadas neste ambiente (Telecontrans, 1999).

A localização geográfica de uma plataforma logística deve contemplar a região onde a mesma está inserida, pois é necessário avaliar as relações provenientes da utilização desta estrutura, isto no âmbito regional, nacional e até mesmo internacional. O seu posicionamento pode ser considerado como estratégico e deve possibilitar a interligação entre os diversos eixos e estar inserida em um meio social e econômico favorável.

Constata-se que existe uma tendência crescente de consolidação deste tipo de empreendimento pelo mundo, muito em razão das vantagens competitivas que podem ser estabelecidas devido a sua diversificada infraestrutura, ao amplo número de atividades e serviços ofertados e também em função do seu posicionamento estratégico (Duarte, 2004; Carvalho, 2010; Rosa, 2005; Dias et al. 2009).

As plataformas logísticas podem colaborar para minimizar os diferentes impactos causados nos centros urbanos pela frequente utilização de veículos de cargas de forma não otimizada ou mesmo sem o planejamento adequado (Cambra-Fierro e Ruiz-Benitez, 2009; Trappey et al. 2011).

As plataformas logísticas podem ser consideradas empreendimentos logísticos complexos onde são desenvolvidas diversas atividades. Apresenta-se uma síntese das principais características deste tipo de empreendimento logístico (Tsamboulas et al. 2003; Wagner, 2010; Hesse e Rodrigue, 2004; Ballis e Mavrotas, 2007): 
- Centralização de diversas atividades logísticas (armazenagem, movimentação, transportes, reembalagem, dentre outras);

- Desenvolvimento de atividades industriais;

- Arranjos que apresentam qualidade nas instalações, oferecendo diversos serviços aos participantes (inquilinos, proprietários, parceiros);

- Ambientes que centralizam e fazem a gestão logística das organizações, tendo flexibilidade para responder as variações de demanda do mercado;

- Localizadas de forma estratégia ao longo da cadeia de suprimentos para obtenção de um menor custo das transações e melhores níveis de serviço;

- Ambientes delimitados capazes de agregar valor aos produtos e aos serviços logísticos prestados;

- Centro de integração de diversos modais de transportes, proporcionando uma otimização de fretes;

- Contribuem para o desenvolvimento social, político e econômico das regiões onde estão instaladas (redução dos níveis de congestionamentos; geração de empregos; utilização racional dos recursos naturais dentre outros).

Entende-se que a utilização de plataformas logísticas, possibilitam as organizações um redesenho de suas estratégias e arranjos logísticos em busca de uma maior eficiência local e global e por apresentarem estas características, os aspectos relacionados a governança destes empreendimentos requerem uma maior atenção.

A governança é composta por procedimentos associados à tomada de decisões, desempenho e controle das organizações e ambientes, com o fornecimento de estrutura capaz de possibilitar uma direção geral para a organização e também satisfazer expectativas razoáveis da prestação de contas para os que estão dentro ou são externos a própria organização (Albers, 2005; Peck et al. 2004). Na seção seguinte, discute-se os principais conceitos relacionados à governança da cadeia de suprimentos, ambiente este onde estão inseridas as plataformas logísticas. 


\subsection{Governança}

A mudança dos padrões de competitividade tem conduzido à formação de diversos tipos de arranjos logísticos na cadeia de suprimentos, podendo ser entre empresas e entre grupos de empresas, os quais podem proporcionar economias de escala e escopo, bem como a aquisição de competências (Albers, 2005; Nassimbeni, 1998; Besanko et al. 2006).

O desenvolvimento e a organização da cadeia de suprimentos são influenciados fortemente pelo grau de relacionamento entre os atores que nela estão inseridos, necessitando de ações coordenadas por parte de seus membros. Para Lambert et al. (1998), na medida em que o relacionamento entre as organizações não é um evento isolado entre as organizações com o mesmo poder de influência e decisão sobre assuntos inseridos no objetivo maior da cadeia de suprimentos ocorre uma variação na assimetria do poder, que influencia e é influenciada pelo modelo de governança existente.

Através das pesquisas realizadas, constata-se que as plataformas logísticas são uma parte importante da cadeia de suprimentos, pois inter-relacionam diferentes atores e desenvolvem um papel relevante tanto em nível operacional como também estratégico das organizações participantes desta mesma cadeia, sendo necessário estabelecer mecanismos de governança adequados a este tipo de ambiente e empreendimentos logísticos.

Uma definição que ajuda a reforçar este pensamento é apresentada por Abrahamsson et al. (2003), onde definem a plataforma logística como uma parte homogênea do sistema de logística, onde uma organização logística estabelece a gestão centralizada e tem a possibilidade de projetar a maneira com qual uma base de recursos possa alcançar novas posições no mercado onde atuam. Para os autores uma plataforma logística inclui ainda conceitos de operações de logística, estrutura física, processos e suas atividades e também deve prover um adequado sistema de informação para suporte na gestão destes empreendimentos.

Para Heide (1994) a governança pode ser compreendia como uma forma de organizar transações ou, de uma forma mais robusta, como um modelo estrutura de coordenação das relações entre os diversos agentes econômicos, no qual contratos de relação são estabelecidos, 
negociados, monitorados, compreendendo um fenômeno de múltiplas dimensões e de um relacionamento duradouro.

O conceito de governança possui múltiplas interpretações, traduzidas por diferentes perspectivas teóricas. Na revisão de literatura sobre governança foram identificados diversos trabalhos que abordam o tema, porém o foco desta pesquisa relaciona a plataforma logística enquanto ambiente ou mesmo arranjo logístico e não somente como uma entidade jurídica. Neste sentido percebe-se que as PL's estão inseridas no âmbito da cadeia de suprimentos, e partindo deste pressuposto realizou-se uma pesquisa de trabalhos que apresentassem contribuições teóricas sobre a governança na cadeia de suprimentos e que os resultados encontrados pudessem auxiliar na elaboração de construtos para o entendimento sobre a governança de plataformas logísticas.

\subsubsection{Conceitos de Governança na Cadeia de Suprimentos aplicáveis a PL's}

Um dos estudos seminais sobre governança é apresentado por Williamson $(1991,1999)$ que aborda a teoria dos custos de transação (TCT), onde o objetivo é explicar qual a instituição será escolhida para governar certa interação econômica. Estas interações podem ocorrer dentro de firmas (relações intraorganizacionais) ou entre firmas (relações interorganizacionais) sendo esta última como mecanismos de governança, isto é, instituições encarregadas pela geração de renda a partir de interdependências entre indivíduos e entre organizações (Ring et al. 2008). Os custos de transação estão relacionados as despesas que os agentes econômicos enfrentam quando compram e vendem no mercado, ou seja, são os custos decorrentes da negociação, elaboração e garantia de cumprimento de um contrato.

Para Williamson (1991), há três estruturas alternativas de governança, o mercado, híbridas e hierárquicas, sendo que as híbridas consistem em contratos e empreendimentos conjuntos. As transações de mercado são governadas pela lei contratual clássica. Nos mercados a identidade das partes é irrelevante e as transações são governadas por termos formais que são fortalecidos por meios legais. A identidade das partes é mais importante em formas híbridas de governança que são apoiadas pela lei neoclássica contratual que é mais adaptável, pois esta forma de governança é caracterizada pela adaptação mútua e maior flexibilidade do que no caso de mercados e há um recurso maior para uma mediação mais flexível para resolução em 
casos de conflitos. A hierárquica tem ainda mais propriedades adaptativas porque ela pode resolver conflitos através da sanção.

A escolha da forma de governança é realizada de acordo com aquele modelo que minimiza não somente os custos de produção, como também os custos de transação. Os custos de transação elevados podem significar ineficiência de utilização do mercado, podendo nestes casos optar por formas hibridas ou hierárquicas (Ring et al. 2008).

Williamson (1999) define a estrutura de governança como um mecanismo de adaptação diante da tarefa de executar transações e a estrutura organizacional pode variar de acordo com determinadas características dessas transações. As estruturas de governança propostas por Williamson (1999) necessitam estar alinhadas com as características das transações que são classificadas como; especificidades de ativos, incerteza e frequência:

- A especificidade de ativos pode ser considerada alta quando é custoso reposicionar posteriormente os ativos de apoio a transação para novos usos. Alta especificidade de ativos aumenta os custos de mudança e torna possível que uma parte mais flexível explore uma menos flexível, direcionando as transações com especificidade de ativos média para as formas híbridas, porque estas fornecem garantias contratuais adicionais e disputam mecanismos de resolução, e direciona aquelas com especificidade de ativos alta para a governança de firmas, porque ainda que estejam sujeitas a custos burocráticos altos, são também mais adaptáveis. As transações com especificidade de ativos baixa serão manuseadas por mercados, aquelas com especificidade de ativos média por híbridos, e aquelas com especificidade de ativos alta em firmas;

- Com relação a especificidade incerteza, considera-se que a mesma não tem importância quando a especificidade de ativos é baixa, uma vez que as partes podem adaptar-se a mudanças inesperadas no ambiente mudando de parceiros. Em condições de especificidade de ativos alta, entretanto, há benefícios em continuar a transação e, portanto a governança híbrida é preferida a fim de facilitar a adaptação. A adaptação não pode ser feita unilateralmente como na governança de mercado, nem por medida, como em firmas. A governança de firma é preferível sempre que a especificidade de ativos e a incerteza são ambas altas e a governança de mercado quando a especificidade de ativos é baixa e a incerteza é alta. 
- Na especificidade frequência o custo de ajuste de híbridos e firmas é pago pelos que efetuam a transação, porém isto possivelmente se torna econômico somente para transações recorrentes.

Segundo Williamson (1991), as transações frequentes que envolvam incertezas sobre seus resultados e requerem investimento em ativos específicos, apresentam uma tendência a ser internalizadas pela firma (hierárquica). Com relação as transações simples, que não apresentam um alto grau de repetição e que não requerem investimento em ativos específicos, deverão ocorrer por meio do mercado (transações com o mercado).

Algumas variáveis como o comportamento oportunista, a racionalidade limitada e a incapacidade dos atores em elaborar contratos que possam contemplar todas as contingências prováveis, associadas às transações, são motivos importantes que levam a firma a preferir internalizar as atividades e, assim, evitar os custos associados à transação de mercado. Além de mercado e hierárquica, há formas alternativas de coordenação da atividade econômica que não podem ser asseguradas nem pela firma, nem pelo mercado, e decorrem da colaboração entre firmas (Williamson 1999; Ring et al. 2008).

Outro ponto abordado por Williamson (1991, 1999), esta relacionado a frequência que caracteriza a recorrência de uma transação. $\mathrm{O}$ aumento da frequência de transação pode ser acompanhado pela elaboração de contratos mais complexos sob forma de reduzir o comportamento oportunista. Em contrapartida, em função do maior envolvimento, em geral e um maior comprometimento dos participantes, acaba gerando uma relação de parceria e dependência bilateral, proporcionando acordos que possam comprometer as transações futuras, resultando em perdas entre as partes. Neste contexto se torna imperativa a necessidade de desenvolver ações de confiança mútua entre as partes envolvidas nas transações recorrentes (Williamson, 1999; Ring et al. 2008).

Para Storper e Harrison (1991), a governança pode ser compreendida como um processo contínuo para acomodar os diversos interesses e realizações cooperativas, pois inclui as relações formais, de hierarquia, poder e obediência, como também, acordos informais, colaborativos em relações não hierárquicas. 
Storper e Harrison (1991) discutem a governança sob a ótica das diferentes relações de poder estabelecidas entre as empresas envolvidas nos arranjos. Os autores utilizam-se de dois conceitos, sendo o core que representa uma situação onde o poder é assimétrico, no qual uma empresa pode determinar a existência das demais e o ring onde o poder é simétrico, aquele em que a existência de uma empresa não é determinada pela tomada de decisões por parte de outra organização. Para os autores, as relações de poder são estabelecidas pela quantidade de atores potenciais envolvidos na cadeia de suprimentos. Storper e Harrison (1991) classificam a governança sob quatro aspectos:

- All ring, no core: As empresas tem o poder simétrico, onde não há exercício de liderança hierárquica, mas sim uma colaboração e simetria entre as partes envolvidas;

- Core-ring (core-ring with - coordinating firm): Tem-se neste caso a presença de uma empresa coordenadora, onde uma empresa pode influenciar as operações internas de outras empresas participantes da cadeia. Existe certo grau de dependência da empresa que tem o poder, pois ela depende das demais para complementar o seu processo;

- Core-ring: Tem-se neste caso a presença de uma empresa condutora, onde uma empresa pode independente de seus parceiros (fornecedores) reestruturar ao menos uma parte da sua rede. A empresa condutora é a parte dominante, exercendo uma hierarquia sobre os envolvidos, tendo o poder assimétrico;

- All-core, no ring: Existe uma empresa verticalmente integrada, onde a dinâmica da governança pode ser alterada em razão da estrutura das empresas ou mesmo da própria organização do sistema.

A abordagem sobre governança, definida por Storper e Harrison (1991) pode ser aplicada na cadeia de suprimentos e em plataformas logísticas, pois permite a construção de alguns pressupostos de como uma cadeia pode ser organizada.

Humphrey e Schimitz (2000) destacam que existem diversas formas de governança, local, pública e privada, as quais exercem um papel importante para o fomento da competitividade dos produtores aglomerados. Os benefícios da aglomeração de empresas não se restringem as economias externas geradas pelas ações dos agentes locais no incentivo as atividades produtivas e no estímulo a rápida difusão do conhecimento. De acordo com Humphrey e 
Schmitz (2000), a governança se relaciona com a coordenação das atividades econômicas, tendo as relações classificadas em:

- Relações com o mercado: o comprador e o produtor não desenvolvem a colaboração entre si na definição do produto, ou mesmo o produto não é padrão. Os riscos podem ser considerados baixos para o comprador. O conhecimento dos compradores sobre o local surge através da reputação dos produtores ou de um fabricante em particular;

- Redes: são formadas entre as firmas de maior ou menor poder semelhante. O relacionamento se estabelece de forma reciproca, objetiva e de complementariedade para obter economias de escopo e escala;

- Quase-hierarquia: onde as firmas, tem uma relação de subordinação como se fosse uma subcontratação. As partes se relacionam no longo prazo e uma assume claramente o papel de liderança;

- Hierarquia: a relação entre as firmas ocorre pelo poder de posse, ficando uma das partes subordinada a outra em sua totalidade. A empresa líder define as regras, o produto e suas especificações e age, em muitos casos como se fosse proprietária dos elos produtivos.

Para Humphrey e Schmitz (2000), a governança se refere a relações entre empresas e os mecanismos institucionais através dos quais se consegue a coordenação extra mercado das atividades dentro de uma cadeia.

Segundo Gereffi $(1994,2000)$ a organização de uma cadeia produtiva global, pode ser caracterizada como a cadeia orientada pelo agente produtivo, a cadeia orientada pelo agente comprador e a cadeia orientada pela informação e neste sentido emerge uma discussão sobre o conceito de governança como uma relação de autoridade e poder capaz de determinar a alocação e os fluxos de recursos financeiros, materiais, humanos e tecnológicos ao longo da cadeia, entre os agentes econômicos envolvidos. Conforme Gereffi (1994, 2000), a organização da cadeia produtiva global compreende as seguintes orientações: 
- Cadeia orientada pelo agente comprador: a função de coordenação do sistema produtivo é exercida por grandes empresas comerciais, que não detêm obrigatoriamente os ativos envolvidos no processo produtivo e que estão geralmente dispersos em diferentes países;

- Cadeia orientada pelo agente produtivo: são aquelas em que geralmente os grandes fabricantes transacionais, realizam a coordenação central das cadeias produtivas, na relação montante e jusante;

- Cadeia orientada pela informação: apresenta uma habilidade em criar mercados em escala e de forma eficiente. Utiliza-se de uma estratégia puxada de negócios radical, o qual substitui as informações de estoques e embarque de produtos para uma situação de demanda real dos consumidores.

Conforme Gereffi, Humphrey e Sturgeon (2005), o processo de descentralização ou mesmo de fragmentação da cadeia de valor em diversos agentes, pressupõe um modelo de governança que seja capaz de fazer a gestão das competências dispersas entre os envolvidos da cadeia, preservando para a empresa líder as atividades consideradas críticas. Para os autores, os elementos que determinam o modelo de governança da cadeia de valor podem ser agrupados em três variáveis:

- Complexidade das transações: esta relacionada a complexidade dos processos de transferência de conhecimento e de informações necessárias para efetivar as transações inerentes à cadeia, com ênfase nas informações específicas de processos e produtos;

- Habilidade de codificar as transações: estabelece o grau em que o conhecimento e as informações podem ser codificadas e transmitidas de forma eficiente entre os envolvidos;

- Competência na cadeia de fornecimento: é a capacidade dos fornecedores atuais e também potenciais em atender os requerimentos da transação.

As variáveis apresentadas influenciam o modelo de governança, os quais de forma evolutiva podem ser compreendidos como: mercado, cadeia de valor modular, cadeia de valor 
relacional, cadeia de valor cativa e cadeia de valor hierárquica. A relação de poder entre os envolvidos na cadeia, é um elemento fundamental para o entendimento do modelo de governança. De acordo com Gereffi et al. (2005), a classificação dos modelos de governança são:

- Mercado: entende-se que as transações comerciais tem como foco a concretização de uma determinada operação e não necessitam ser obrigatoriamente transitórias e finitas entre si. As relações estabelecidas podem ser transacionais e ocorrerem por diversas vezes, sem, contudo que sejam estabelecidas parcerias que extrapolem a transação entre os membros;

- Cadeia de valor modular: aplica-se a cadeias que tem como características a produção ou mesmo na constituição de seus produtos à arquitetura modular, nas quais padrões técnicos pré-acordados podem reduzir as interações entre os módulos que constituem o produto final, reduzindo desta forma a variabilidade de cada módulo e consequentemente o resultado da cadeia;

- Cadeia de valor relacional: as interações entre as organizações mostram-se complexas, onde existe a dependência mútua e níveis elevados de especificidades dos ativos. Nota-se nestes casos um maior grau de dependência do compartilhamento de conhecimento tácito e de ativos específicos entre os agentes da cadeia;

- Cadeia de valor cativa: neste caso, existe uma diferença na relação de poder entre as partes que compõe a cadeia de valor, onde há um grau de dependência de alguns agentes por algum outro membro que detêm o poder. Os riscos e custos mostram-se elevados em caso de mudança de determinados participantes. Os agentes que compõe este modelo, geralmente possuem competências restritas em atividades específicas, produtos ou serviços que demandam uma maior complexidade de execução;

- Cadeia de valor hierárquica: este modelo de governança se caracteriza pela integração vertical entre os agentes que compõe a cadeia de valor de determinado produto ou serviço, onde se tem a existência de acordos pré-determinados e claros de subordinação entre os agentes. 
$\mathrm{Na}$ abordagem dos modelos de governança propostos por Gereffi et al. (2005), é possível perceber a necessidade e o dinamismo que a governança deve proporcionar em razão das alterações que podem ocorrer no ambiente ou mesmo dos agentes participantes da cadeia. Verifica-se que os modelos propostos aplicam-se também à governança da cadeia de suprimentos e das plataformas logísticas.

De acordo com Jessop (1998), a governança pode ser vista sob dois aspectos, sendo o primeiro refere-se a qualquer modo de coordenação das atividades interdependentes, sendo classificados como a anarquia de trocas (mercado), hierarquia organizada e heterquia que se auto-organiza. No segundo aspecto, mais restrito o autor considera que a auto-organização pode se dar de três formas:

- Conexão interpessoal: a forma na qual as pessoas representam a si próprio ou seus sistemas funcionais, mas não compete a estas pessoas o poder de representação dos organismos ou organizações;

- Auto-organização de relações entre as organizações: percebe-se a existência de coordenação e negociação em torno dos benefícios e interesses mútuos, nos quais se faz necessário o controle de recursos interdependentes;

- Direcionamento entre os sistemas: consiste em uma organização norteada para determinadas missões, formada por sistemas funcionais que não tem autonomia para controlar o desenvolvimento geral da economia, mas podem realizar intervenções na mesma em aspectos que sejam de seu domínio ou competência.

As três formas de governança por uma auto-organização estão conectadas, pois a confiança interpessoal pode facilitar a negociação entre as empresas e o diálogo entre as mesmas facilita a comunicação entre o sistema. Para o autor, a definição dos níveis de eficiência da governança não é uma tarefa fácil, pois não há um conjunto de critérios prontos que permita testar o sucesso da governança. Em muitos casos o fracasso da governança esta relacionado a incapacidade presumível de fazer um novo planejamento dos objetivos frente a um desacordo persistente, bem como a validação das metas para os diferentes agentes que intervém de alguma forma no resultado (Jessop, 1998). 
A função básica do sistema de governança é coordenar as atividades dos diferentes atores envolvidos em uma relação de coordenação, bem como motivar e incentivar os participantes a cumprir os termos do acordo constituído. A coordenação é definida como o processo de organização ou de adaptação de componentes individuais em uma determinada ordem. A fim de avaliar prováveis diferenças entre os comportamentos adequados e inadequados, o sistema de governança deve prover formas de controle ou monitoramento das atividades, onde o controle pode ser entendido como o processo de monitoramento e avaliação do desempenho dos diversos intervenientes (Roth et al. 2010; Albers, 2005).

\section{Plataformas Logísticas na Europa}

As plataformas logísticas são empreendimentos logísticos de grande porte que atendem a uma área ou região, onde se concentram e realizam-se atividades voltadas a produção, transportes, logística e distribuição de mercadorias, utilizando-se de uma mesma base de serviços capazes de inter-relacionar diversos atores da cadeia de suprimentos, sejam estes públicos ou privados.

De acordo com Colin (1996), a Europa passou a demonstrar uma preocupação com relação aos arranjos logísticos existentes, isto no âmbito nacional como regional, passando a idealizar localizações que pudessem possibilitar um melhor nível de serviço e por consequência uma redução dos custos ao longo da cadeia logística.

Com base em pesquisas sobre as características de plataformas logísticas na Europa desenvolvidas por Boile et al. (2009) e Higgins e Ferguson (2011) e expressas nos relatórios Feasibility of Freight Villages in the NYMTC Region e An Exploration of the Freight Village Concept and its Applicability to Ontario, foi elaborado uma síntese das principais características destes empreendimentos.

O objetivo deste levantamento das características, a fim de que as mesmas possam contribuir para uma discussão sobre os aspectos gerais de governança nas plataformas logísticas à luz dos modelos de governança na cadeia de suprimentos apresentados anteriormente. Na Tabela 1, demonstra-se uma síntese dos resultados extraídos dos relatórios: 
Tabela 1 - Síntese das características das Plataformas logísticas na Europa ${ }^{2}$

\begin{tabular}{|c|c|c|c|c|c|}
\hline \multicolumn{2}{|c|}{ Elementos/Características das PL's } & $\begin{array}{l}\text { Roissy } \\
\text { Sogaris }\end{array}$ & $\begin{array}{c}\text { Nordic } \\
\text { Transport } \\
\text { Centre } \\
\text { (NTC) } \\
\end{array}$ & $\begin{array}{c}\text { GVZ } \\
\text { Bremen }\end{array}$ & $\begin{array}{c}\text { Interporto } \\
\text { Bologna }\end{array}$ \\
\hline \multirow{11}{*}{ Objetivos } & Mitigar o congestionamento & $\checkmark$ & & & \\
\hline & $\begin{array}{l}\text { Promover o transporte } \\
\text { intermodal }\end{array}$ & $\checkmark$ & & & \\
\hline & Suporte para os negócios & $\checkmark$ & $\checkmark$ & & \\
\hline & $\begin{array}{c}\text { Realocar o frete em } \\
\text { instalações fora das cidades }\end{array}$ & & $\checkmark$ & $\checkmark$ & \\
\hline & $\begin{array}{l}\text { Melhoria do Ambiente e } \\
\text { segurança p/atividades }\end{array}$ & & $\checkmark$ & & \\
\hline & Reduzir o congestionamento & & & $\checkmark$ & \\
\hline & $\begin{array}{c}\text { Consolidar as atividades da } \\
\text { indústria }\end{array}$ & & & $\checkmark$ & $\checkmark$ \\
\hline & $\begin{array}{l}\text { Racionalização de utilização } \\
\text { da terra }\end{array}$ & & & $\checkmark$ & \\
\hline & $\begin{array}{c}\text { Promoção de } \\
\text { desenvolvimento regional }\end{array}$ & & & $\checkmark$ & \\
\hline & $\begin{array}{c}\text { Apoiar/suporte na } \\
\text { competitividade intermodal }\end{array}$ & $\checkmark$ & $\checkmark$ & & $\checkmark$ \\
\hline & $\begin{array}{l}\text { Capac. de distribuição para os } \\
\text { centros urbanos }\end{array}$ & & & $\checkmark$ & $\checkmark$ \\
\hline \multirow{2}{*}{ Gestão } & Privada/pública & Sogaris & $\begin{array}{l}\text { The NTC, } \\
\text { Ltd. }\end{array}$ & $\begin{array}{r}\text { GVZE } \\
\text { Bremen }\end{array}$ & $\begin{array}{l}\text { Bologna } \\
\text { SPA }\end{array}$ \\
\hline & $\begin{array}{l}\text { Constituição - Parceria } \\
\text { Público-Privada }\end{array}$ & $\checkmark$ & $\checkmark$ & $\checkmark$ & $\checkmark$ \\
\hline \multirow{4}{*}{$\begin{array}{l}\text { Informações } \\
\text { Gerais }\end{array}$} & Atividade Industrial & $\checkmark$ & $\checkmark$ & $\checkmark$ & $\checkmark$ \\
\hline & QT de empresas & 100 & 15 & 150 & 81 \\
\hline & QT de Empregados & 2500 & - & 8000 & 1500 \\
\hline & Área $\left(\mathrm{m}^{2}\right)$ & 550.000 & 592.000 & 3.600 .000 & 2.000 .000 \\
\hline
\end{tabular}

De acordo com Boile et al. (2009) e Higgins e Ferguson (2011), as plataformas logísticas da Europa (Tabela 1) apresentam algumas similaridades de características e objetivos entre si, dentre as quais é possível destacar:

- Reduzir os congestionamentos nos centros urbanos;

- Melhoria do ambiente e desenvolvimento local e regional;

- Utilizam-se da intermodalidade, com ênfase nos modais ferroviária e rodoviário;

\footnotetext{
${ }^{2}$ Fonte: Adaptado de Boile et al. (2009) e Higgins e Ferguson (2011).
} 
- A organização institucional dos empreendimentos em sua maioria é realizado sob a forma de uma parceria público-privada (PPP);

- Existe um grande número de empresas e também de empregados participantes das plataformas logísticas;

- São realizadas atividades industriais nestes empreendimentos e desta forma agregam valor na cadeia de suprimentos;

- A área onde localizam as PL's são de grandes extensões, ficando geralmente afastadas dos centros urbanos.

Conforme demonstrado na Tabela 1 aproximadamente de $80 \%$ das plataformas logísticas pesquisadas tem o formato de constituição organizacional baseado na parceria públicoprivada, pois os riscos financeiros podem ser compartilhados entre os participantes do setor privado e público.

No âmbito público, pode-se fornecer a infraestrutura necessária de transporte e serviços públicos e fornecer uma variedade de incentivos, tais como redução de impostos e arrendamentos a longo prazo para atrair empresas, enquanto o setor privado pode fornecer o capital para a construção de terminais, armazéns, centros de distribuição e outras instalações relacionadas (Boile et al. 2009; Higgins e Ferguson, 2011; Kapros, 2011).

Uma estrutura adequada de governança e um plano sustentável de gestão pode contribuir para assegurar o funcionamento das plataformas logísticas. Para Leitner e Harrison (2001), o desenvolvimento de uma plataforma logística não pode atingir o sucesso sem o financiamento do capital, ações de marketing e da cooperação entre os setores público e privado.

Dentre os diferentes modelos de governança da cadeia de suprimentos apurados anteriormente, percebe-se que a governança das plataformas logísticas Europeias da Tabela 2 indicam uma possível aderência aos modelos de governança híbrida, proposta por Humphrey e Schmitz (2000), Gereffi et al. (2005), Storper e Harrison (1991) e Jessop (1998) sintetizados na Tabela 2: 
Tabela 2 - Atributos de governança aplicáveis a plataformas logísticas ${ }^{3}$

\begin{tabular}{|c|c|c|}
\hline $\begin{array}{c}\text { Plataformas } \\
\text { Logísticas }\end{array}$ & Autores & Síntese dos atributos dos modelos de governança \\
\hline & $\begin{array}{c}\text { Humphrey e } \\
\text { Schmitz (2000) }\end{array}$ & $\begin{array}{l}\text { *Redes: são formadas entre as firmas de maior ou } \\
\text { menor poder semelhante. O relacionamento se } \\
\text { estabelece de forma reciproca e objetiva e de } \\
\text { complementariedade para obter economias de } \\
\text { escopo e escala; } \\
\text { *Quase-hierarquia: onde as firmas, tem uma relação } \\
\text { de subordinação como se fosse uma subcontratação } \\
\text { As partes se relacionam no longo prazo e uma } \\
\text { assume claramente o papel de liderança. }\end{array}$ \\
\hline Roissy Sogaris & $\begin{array}{l}\text { Gereffi, Humphrey } \\
\text { e Sturgeon (2005) }\end{array}$ & $\begin{array}{l}\text { * Cadeia de valor modular: aplica-se a cadeias que } \\
\text { tem como características a produção ou na } \\
\text { constituição de seus produtos de arquitetura } \\
\text { modular, nas quais padrões técnicos pré-acordados } \\
\text { podem reduzir as interações entre os módulos que } \\
\text { constituem o produto final, reduzindo desta forma a } \\
\text { variabilidade de cada módulo e consequentemente o } \\
\text { resultado da cadeia. }\end{array}$ \\
\hline \multirow[t]{2}{*}{$\begin{array}{c}\text { Nordic Transport } \\
\text { Centre (NTC) } \\
\text { GVZ Bremen } \\
\text { Interporto Bologna }\end{array}$} & $\begin{array}{c}\text { Storper e Harrison } \\
\text { (1991) }\end{array}$ & $\begin{array}{l}\text { * Core-ring (core-ring with -coordinating firm) } \\
\text { Tem-se neste caso a presença de uma empresa } \\
\text { coordenadora, onde uma empresa pode influenciar } \\
\text { as operações internas de outras empresas } \\
\text { participantes da cadeia. Existe certo grau de } \\
\text { dependência da empresa que tem o poder, pois ela } \\
\text { depende das demais para complementar o seu } \\
\text { processo; } \\
\text { * Core-ring: Tem-se neste caso a presença de uma } \\
\text { empresa condutora, onde uma empresa pode } \\
\text { independente de seus parceiros (fornecedores) } \\
\text { reestruturar ao menos uma parte da sua rede. A } \\
\text { empresa condutora é a parte dominante, exercendo } \\
\text { uma hierarquia sobre os envolvidos, tendo o poder } \\
\text { assimétrico. }\end{array}$ \\
\hline & Jessop (1998) & $\begin{array}{l}\text { *Auto-organização de relações entre as } \\
\text { organizações: percebe-se a existência de } \\
\text { coordenação e negociação em torno dos benefícios } \\
\text { e interesses mútuos, nos quais se faz necessário o } \\
\text { controle de recursos interdependentes; }\end{array}$ \\
\hline
\end{tabular}

Entende-se que os atributos de governança identificados neste trabalho podem servir de indícios (não conclusivos) ou mesmo fornecer um caminho para o entendimento sobre a governança em plataformas logísticas, pois nos diversos trabalhos pesquisados verificou-se

\footnotetext{
${ }^{3}$ Fonte: Elaborado pelos autores.
} 
que este tema não apresenta ainda uma abordagem mais específica, sendo dada uma ênfase nas discussões sobre a gestão dos empreendimentos, isto é, uma dimensão intraorganizacional e não no sentido das relações entre as organizações inseridas nas plataformas logísticas que atuam como elos da cadeia de suprimentos.

Um dos principais aspectos a ser considerados na organização das plataformas logísticas é o relacionamento entre os diversos atores envolvidos neste tipo ambiente, pois a cooperação entre as partes pode se dar de maneira horizontal ou vertical e os objetivos devem estar alinhados. $\mathrm{O}$ entendimento e análise dos modelos de governança aplicáveis nas plataformas logísticas são de grande importância para determinar não só de que maneira estão distribuídas as atividades econômicas neste ambiente, mas também evidenciar as relações entre as partes, o trade-off relacionado aos ganhos reais e potenciais e também contribuir para desenvolvimento destes empreendimentos no cenário atual, seja este local ou global.

\section{Análise e discussões dos resultados da pesquisa}

Em análise aos modelos de governança aplicados a cadeia de suprimentos elucidados durante a construção do referencial teórico e discutidos durante a pesquisa, constata-se que alguns atributos e elementos destes modelos podem ser correlacionados a governança das plataformas logísticas. Nesta seção são discutidos os resultados encontrados na pesquisa, considerando o referencial teórico pesquisado e também as práticas de plataformas logísticas estudadas.

A governança de plataformas logísticas em diversos trabalhos pesquisados converge para uma discussão no campo da gestão das operações das plataformas logísticas, onde tratam as questões relacionadas à gestão dos recursos, sejam estes físicos, humanos, financeiros dentre outros, porém é necessário estabelecer uma distinção entre gestão e governança, pois a governança trata de aspectos relacionados a outras dimensões das relações como o poder, a hierarquia entre os diferentes atores que estão envolvidos ou participam de um mesmo ambiente ou são impactados pelas ações decorrentes destas estruturas.

Em uma plataforma logística são estabelecidos diferentes tipos de relações entre os atores envolvidos, as quais devem ser desenvolvidas de forma conjunta, onde objetivos necessitam 
estar alinhados para a obtenção de uma maior sinergia. Os benefícios sinérgicos decorrentes deste alinhamento, podem ser desde o compartilhamento e otimização de recursos até o desenvolvimento econômico dos empreendimentos que fazem parte das plataformas logísticas (Higgins e Ferguson, 2011).

Percebe-se que são diversos os atores envolvidos no ambiente das plataformas logísticas, os quais necessitam estabelecer para a sua própria "manutenção de existência" uma relação justa entre as partes. A adoção de uma estrutura e mecanismos de governança tornam-se imprescindíveis a estes ambientes. Apresenta-se os principais autores pesquisados que serviram de base caracterização das estruturas dos modelos de governança aplicáveis as plataformas logísticas.

- Williamson (1991,1999): Aborda os fatores levados em consideração na escolha de uma ou mais empresas para transacionar no mercado, os motivos de integra-se de forma verticalizada ou mesmo por adotar determinada estrutura de governança híbrida nas suas relações com outras empresas. No âmbito das plataformas logísticas, a abordagem teórica serve para analisar a forma de decisão e organização de uma ou mais empresas na obtenção de insumos, produção ou distribuição na cadeia de suprimentos, seja através de uma estrutura de mercado, hierarquia ou híbrida;

- Jessop (1998): A discussão sobre governança é realizada no âmbito das relações entre as empresas e o estado, tendo background as relações sociais. As plataformas logísticas utilizam em alguns casos (exemplo Tabela 1) da parceria público-privada (PPP) e a contribuição teórica deste modelo de governança, esta no fornecimento de elementos que contribuam para a compreensão de como as relações de coordenação e negociação entre as partes privada \& pública ocorre em torno dos benefícios e interesses mútuos, nos quais se faz necessário o controle de recursos interdependentes;

- Gereffi (1994, 2000): A abordagem do autor esta centrada nas discussões sobre as cadeias globais de valor, analisando os elos capazes de determinam ou influenciar o desempenho dessas cadeias, sejam elas formadas por produtores, compradores ou mesmo a informação que pode atuar na condução da cadeia. Algumas plataformas logísticas atuam no âmbito global, ou mesmo como elo de informação entre os atores logísticos pertencentes a uma mesma cadeia, conforme a abordagem de Aldin e Stahre 
(2003) e Abrahamsson et al. (2003). As plataformas logísticas compreendem o desenvolvimento de atividades industriais e logísticas, que se inter-relacionam adicionando valor aos produtos e serviços;

- Humphrey e Schmitz (2000): Os autores estabelecem que independentemente das cadeias serem conduzidas pelos produtores ou por compradores, podem ser desenvolvidas diversas formas de relacionamento entre as partes e em decorrência disto adota-se uma estrutura de governança que atenda aos objetivos propostos. As plataformas logísticas são ambientes compostos por diversos agentes, os quais se articulam em razão dos objetivos que se estabelecem e necessitam de estruturas de governança adaptáveis a realidade do ambiente em que estão inseridos;

- Storper e Harrison (1991): Discutem a governança sobre o prisma de um dos seus principais elementos que é o poder. Em empreendimentos como as plataformas logísticas que atuam como parte da cadeia de suprimentos, a abordagem feita pelos autores corrobora para o entendimento de como a cadeia esta organizada e a intensidade do poder exercidos pelo diferentes atores envolvidos. Os conceitos abordados pelos autores permite uma análise de parte da cadeia, não envolvendo apenas pares de empresas, mas um número maior de níveis relacionais entre os participantes.

- Gereffi, Humphrey e Sturgeon (2005): Na abordagem dos modelos de governança propostos pelos autores, é possível perceber a necessidade e o dinamismo que a governança deve proporcionar em razão das alterações que podem ocorrer no ambiente ou mesmo dos agentes participantes da cadeia. Verifica-se que os modelos propostos aplicam-se também à governança de plataformas logísticas.

Sintetizando as abordagens apresentadas e discutidas neste trabalho, percebe-se que as características e elementos contemplados nos modelos de governança da cadeia de suprimentos apresentam uma aderência a governança de plataformas logísticas, possibilitando, desta maneira, uma contribuição no âmbito acadêmico e profissional sobre as relações interorganizacionais que se estabelecem nestes empreendimentos, muitas vezes exercendo um grau de influência e hierarquia entre os participantes. Neste mesmo sentido, Meiduté (2007), recomenda que as plataformas logísticas sejam geridas por uma entidade 
jurídica neutra, possibilitando assim uma maior sinergia e cooperação entre as partes envolvidas.

A cooperação mútua entre diferentes participantes de uma plataforma logística é um ponto que deve ser constantemente trabalhado, pois é difícil para os envolvidos planejarem suas atividades sob forma a atender não somente aos seus próprios interesses, mas também aos objetivos dos demais integrantes destes empreendimentos. Os obstáculos mais comuns para a cooperação entre os participantes de plataformas logísticas são (Cruijssen et al. 2007):

- Atores/Participantes com diferentes objetivos - Ausência de objetivos comuns;

- Comportamento oportunista entre os envolvidos (abordagem de Williamson, 1991,1999);

- Baixo grau de confiabilidade entre os parceiros para liderar a cooperação;

- Custos elevados pela falta de sincronismo em procedimentos estratégicos e operacionais;

- Ausência de diferenciação dos produtos ou serviços perante os clientes em razão da baixa sinergia nos processos dentre outros aspectos.

As plataformas logísticas são empreendimentos complexos e diversificados, tanto no que concerne a produtos, participantes, fluxo de mercadorias, infraestrutura disponível, serviços oferecidos, impactos econômicos e sociais causados na região em que se estão localizadas dentre outros aspectos, o que conduzem e reforçam a ideia de que é necessário uma compreensão dos aspectos relacionados a governança exercida nestes ambientes.

Para uma análise da governança em plataformas logísticas e tomando como base os referenciais teóricos e também os casos de PL's Europeias verificados nos relatórios técnicos Feasibility of Freight Villages in the NYMTC Region e An Exploration of the Freight Village Concept and its Applicability to Ontario (Boile et al. 2009; Higgins e Ferguson, 2011) foi elaborado a Tabela 3 onde sugere-se alguns atributos e elementos a serem considerados para a estruturação e orientação do modelo de governança nestes empreendimentos. 
Tabela 3 - Proposição de atributos e elementos que compõe a governança de PL's ${ }^{4}$

\begin{tabular}{|c|c|}
\hline \multicolumn{2}{|c|}{ Governança em Plataformas Logísticas - GPL's } \\
\hline Atributos/Elementos & Descrição \\
\hline Objetivos das Plataformas Logísticas & Identificar quais os objetivos das PL's \\
\hline Organização Institucional & Privada, Pública, Parceria Público-Privada \\
\hline Serviços e Atividades & Apurar quais os serviços e ou atividades realizadas \\
\hline Nível de formalização & 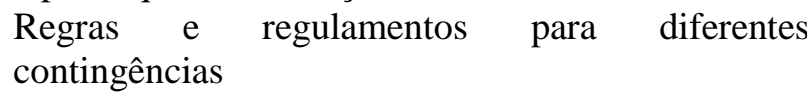 \\
\hline Modelo de gestão & Vertical, Horizontal, Organismos de gestão \\
\hline Participantes/Inquilinos (PL's) & Identificação dos participantes internos das PL's \\
\hline Relações externas & $\begin{array}{l}\text { Identificar os atores externos que mantem } \\
\text { relacionamento com as PL's }\end{array}$ \\
\hline Metas da organização & $\begin{array}{l}\text { Verificar quais as metas estabelecidas pela gestão } \\
\text { do empreendimento }\end{array}$ \\
\hline Especificidades dos ativos e recursos & $\begin{array}{l}\text { Identificar os ativos e recursos que compõe o } \\
\text { empreendimento e o nível de compartilhamento } \\
\text { entre os participantes }\end{array}$ \\
\hline Simetria e Assimetria & $\begin{array}{l}\text { Identificar os níveis de simetria e assimetria entre } \\
\text { os envolvidos }\end{array}$ \\
\hline Fluxo de Informações & $\begin{array}{l}\text { Identificar os canais de informações utilizados } \\
\text { níveis de eficiência }\end{array}$ \\
\hline Indicadores de desempenho & $\begin{array}{l}\text { Verificar os indicadores de desempenho utilizados, } \\
\text { tanto em níveis operacionais como estratégicos. }\end{array}$ \\
\hline Mapeamento da cadeia & $\begin{array}{l}\text { Realização do mapeamento da cadeia de } \\
\text { suprimentos que o empreendimento esta inserido }\end{array}$ \\
\hline Abrangência e Atuação & Local, regional ou global \\
\hline
\end{tabular}

Os atributos e elementos apresentados na Tabela 3 podem contribuir para a busca pelo entendimento sobre a governança em plataformas logísticas. Para Leitner e Harrison (2001), uma estrutura adequada de governança e também uma gestão dos recursos são fatores fundamentais na condução e desenvolvimento de plataformas logísticas.

\footnotetext{
${ }^{4}$ Fonte: Elaborado pelos Autores.
} 


\section{Conclusão}

A fim de atingir o objetivo proposto com esta pesquisa, que foi identificar os atributos e elementos que pudessem ser aplicados à governança das plataformas logísticas realizou-se uma revisão bibliográfica em meios eletrônicos como base de dados, sites de plataformas logísticas e também utilizou-se artigos, teses de doutorado, dissertações de mestrado, livros dentre outros materiais.

Constatou-se ao longo da pesquisa que o tema governança, trata-se de uma nova abordagem dos assuntos já tratados sobre as plataformas logísticas, pois a governança deste tipo de empreendimento deve estar associada a uma discussão sobre a influência do poder, o grau de hierarquia entre os diferentes atores envolvidos ou participantes e também os níveis e sinergias que se estabelecem nas relações interorganizacionais tanto na esfera pública como privada.

Ficou evidenciado durante a pesquisa que as plataformas logísticas apresentam práticas deste tipo de empreendimento mais desenvolvidas na Europa, onde a gestão destes mesmos empreendimentos pode ficar a cargo da iniciativa privada, pública ou em muitos casos na parceria público-privada, e neste sentido o modelo de governança adotado nestes ambientes e os níveis de relações estabelecidas entre os participantes devem atender a objetivos comuns proporcionando benefícios mútuos e vantagens competitivas aos envolvidos. O modelo de governança pode ser considerado como um instrumento fundamental para uma plataforma logística, onde o uso efetivo deste instrumento pode proporcionar uma maior eficiência na gestão dos recursos e também direcionar as competências específicas de cada um dos envolvidos de forma sinérgica.

A partir das contribuições deste estudo sobre os conceitos e elementos da governança da cadeia de suprimentos que tenham aplicabilidade e aderência à governança de plataformas logísticas, entende-se que a governança é composta por procedimentos associados à tomada de decisões, desempenho e controle das organizações, com o fornecimento de estrutura para dar uma direção geral para a organização.

No âmbito acadêmico, são diversas as publicações que abordam o tema plataforma logística, principalmente trabalhos que tem como background as operações realizadas em países 
localizados na Europa. Neste sentido entende-se como oportunidade o desenvolvimento de futuras pesquisas acadêmicas que abordem e discutam os indicadores de desempenho aplicáveis a governança de plataformas logísticas, dada a complexidade deste tipo de empreendimentos logístico, a diversidade de atores envolvidos e os diversos serviços realizados.

Por fim entende-se como contribuição do artigo, os resultados apresentados nesta pesquisa, os quais poderão ser utilizados como hipóteses no desenvolvimento de novas pesquisas que ajudem a refinar e tornar mais robusto o conhecimento que se tem acerca da governança em plataformas logísticas.

\section{Agradecimentos}

Agradecemos ao CNPq - Conselho Nacional de Desenvolvimento Científico e Tecnológico pelo apoio durante a realização desta pesquisa.

\section{Referências}

Abrahamsson, M., Aldin, N. e Stahre, F. (2003) Logistics platforms for improved strategic flexibility. International Journal of Logistics Research and Applications, vol. 6, n. 3, pp. 85-106.

Albers, S. (2005) The design of alliance governance systems. Köln: Kölner Wissenschaftsverlag.

Aldin, N e Stahre, F. (2003) Electronic commerce, marketing channels and logistics platforms - a wholesaler perspective. European Journal of Operational Research, vol. 144, n. 2, pp. 270-279.

Ballis, A. e Mavrotas, G. (2007) Freight village design using the multicriteria method PROMETHEE. Operational Research, vol. 7, n. 2, pp. 213-231.

Besanko, D., Dranove, D., Schaefer, S. e Shanley, M. (2006) A economia da estratégia. Porto Alegre: Bookman $-3^{\mathrm{a}}$ edição.

Boile, M., Theofanis, S. e Gilbert, P. (2009) Task 5: analyzing Freight Village opportunities. Feasibility of freight villages in the NYMTC region, Disponível em http://www.nymtc.org/project/freight_planning/frtvillage/FrtVillage_files - Acessado em agosto de 2012.

Boudoin, D. (1996) Logística-Território-Desenvolvimento: O caso europeu. I Seminário Internacional: Logística, Transportes e Desenvolvimento. Ceará: UFC/CT/DET p. 105.

Cambra-Fierro, J. e Ruiz-Benitez, R. (2009) "Advantages of intermodal logistics platforms: insights from a Spanish platform". Supply Chain Management: An International Journal, vol. 14, n. 6, pp. 418- 421.

Campolongo, M., Morandi, C. e Mariotti, I. (2010) La piattaforma logistica di Leixões, Portogallo, e il suo território. Journal of Land Use, Mobility and Environment, vol. 3, n. 2, pp. 65-72. 
Carvalho, C. C. (2010) Análise de Benchmarking Para Projeto de Plataforma Logística: Caso da Plataforma Logística de Campinas. Dissertação de Mestrado. Programa de Pós-Graduação em Engenharia Civil. Campinas, UNICAMP.

Colin, J. (1996) Les evolutions de la logistique en Europe: vers la polarisation des espaces. I Seminário Internacional: Logística, Transportes e Desenvolvimento. Ceará, pp. 52-92.

Cooper, M. e Ellram, L. (1993) Characteristics of supply chain management and the implications for purchasing and logistics strategy. The International Journal of Logistics Management, vol. 4, n. 2, pp. 13-24.

Creswell, J. W. (2007) Projetos de pesquisa: Métodos qualitativo, quantitativo e misto. Porto Alegre: Artmed - $2^{a}$ edição.

Cruijssen, F., Cools, M. e Dullaert, W. (2007) "Horizontal cooperation in logistics: opportunities and impediments". Transportation Research Part E, vol. 43, n. 2, pp.129-42.

Dias, J. C. Q., Calado, J. M. F., Osório, A. L. e Morgado, L. F. (2009) RFID together with multiagent systems to control global value chains. Annual Reviews in Control, vol. 33, n. 2, pp. 185195.

Duarte, P. C. (2004) Desenvolvimento de um mapa estratégico para apoiar a implantação de uma Plataforma Logística. Tese de Doutorado. Programa de Pós- Graduação em Engenharia de Produção. Porto Alegre. UFRGS.

Dubke, A. F., Ferrera, F. R. N. e Pizzolato, N. D. (2004) Plataforma Logística: características e tendências para o Brasil. XXIV ENEGEP - Florianópolis, SC, Brasil.

Europlatforms EEIG. Logistics Centres Directions for Use (2004) Disponível em: http://www.unece.org - Acesso em 16 de Julho de 2012.

Gajsek, B., Lipicnik, M. e Simenc, M. (2012) The logistics platform disambiguation. Research in Logistics \& Production, vol. 1, n. 2, pp. 69-80.

Gereffi, G. (1994) The organization of buyer-driven global commodity chains: how U.S. retailers shape overseas production networks. In: Gereffi, G. e Korzeniewicz, M. (1994) Commodity chains and global capitalism. Westport: Praeger.

Gereffi, G. (2000) Beyond the producer-driven/buyer-driven dichotomy: an expanded typology of global value chains, with special reference to the Internet. [S.l.: s.n.].

Gereffi, G., Humphrey, J. e Sturgeon, T. (2005) The governance of global value chains. Review of International Political Economy, vol. 12, n. 1, pp. 78-104.

Heide, J. B. (1994) Interorganizational governance in marketing channels. Journal of Marketing, v. 58, n. 2, pp. 71-85.

Hesse, M. e Rodrigue, J. (2004) The transport geography of logistics and freight distribution. Journal of Transport Geography, vol. 12, n. 3, pp. 171-184.

Higgins, C. D. e Ferguson, M. R. (2011) An Exploration of the Freight Village Concept and its Applicability to Ontario. McMaster Institute of Transportation and Logistics. McMaster University. Hamilton, Ontario. October, pp. 195.

Humphrey, J. e Schmitz, H. (2000) Governance and Upgrading: Linking Industrial Cluster and Global Value Chain Research. IDS Working Paper 120.

Jessop, B. (1998) The rise of governance and the risk of failures: the case of economic development. International Social Science Journal, Vol. 1, n.155, pp. 29-45.

Kobayashi, S. (2000) Renovação da logística: como definir as estratégias de distribuição física global. São Paulo: Atlas $-1^{\mathrm{a}}$ edição. 
Lambert, D. M., Stock, J. R. e Ellram, L. M. (1998) Fundamentals of logistics management. New York: McGraw-Hill.

Leitner, S. e Harrison, R. (2001) The Identification and Classification of Inland Ports, Research Report 4083-1, Center for Transportation Research, Texas Department of Transportation, Austin, Texas, August.

Marconi, M. A. e Lakatos, E. M. (2009) Fundamentos de metodologia científica. São Paulo: Atlas $-6^{a}$ edição.

Matera, R. R. T. (2012) O desafio logístico na implantação de um aeroporto indústria no Brasil. Journal of Transport Literature, vol. 6, n. 4, pp. 190-214.

Meidute, I. (2007) Economical evaluation of logistics centres establishment. Transport, vol. 22, n. 2, pp. 111-1117.

Mentzer, J., De Witt, W., Keebler, J., Min, S., Nix, N., Smith, C. e Zacharia, Z. (2001) Defining Supply Chain Management. Journal of Business Logistics, vol. 22, n.2, pp.1-25.

Nassimbeni, G. (1998) Network structures and co-ordination mechanisms: a taxonomy. International Journal of Operations \& Product Management, vol. 18, n. 6, pp. 538-544.

Peck, E., Six, P., Glasby, J. e Skelcher, C. (2004) "Governance and Partnerships". Journal of Integrated Care, vol. 12 n. 4, pp. 3-8.

Pettit, S. J. e Beresford, A. K. C. (2009) Port development: from gateways to logistics hubs. Maritime Policy and Management, vol. 36, n. 3, pp. 253-267.

Pfohl, H. e Buse, H. P. (2000) Inter-organizational logistics systems in flexible production networks. An organizational capabilities perspective. International Journal of Physical Distribution \& Logistics Management, vol. 30, n. 5, pp. 388-408.

Rimienè, K. e Grundey, D. (2007) Logistics Centre Concept through Evolution and Definition. Engineering Economics, vol. 4, pp. 87-95.

Ring, P. S., Huxham, C., Ebers, M. e Cropper, S. (2008) The Oxford Handbook of InterOrganizational Relations. Oxford: Oxford University Press.

Rosa, D. P. (2005) O planejamento de centros logísticos com base na agregação de valor por serviços logísticos em terminais de transporte. Tese de Doutorado. Programa de Pós-Graduação em de Transportes. Rio de Janeiro. COPPE/UFRJ.

Roth, A. L., Wegner, D., Antunes Jr. e Padula, A. D. (2010) Diferenças e inter-relações dos conceitos de Governança e Gestão de Redes Interorganizacionais: contribuições para o campo de estudos. In: Encontro Nacional dos Programas de Pós-Graduação em Administração, Anais.

Storper, M. e Harrison, B. (1991) Flexibility, hierarchy and regional developments: the changing structure of industrial production systems and their forms of governance in the 1990s. Research Policy, vol. 20, n. 5, pp. 407-422.

Telecotrans (1999) Plataformas logísticas y centros de transporte de mercancias em Espana: uma visione de la situación actual y proposta de intevencion. Disponível em http://www.telecotrans.es - Acesso em 08 setembro de 2012.

Trappey, C. V., Linb, G. Y. P., Trappey, A. J. C., Liud, C. S. e Leed, W. T. (2011) Deriving industrial logistics hub reference models for manufacturing based economies. Expert Systems with Applications, vol. 38, n. 2, pp. 1223-1232.

Tsamboulas, D. A. e Kapros, S. (2003) "Freight Village Evaluation Under Uncertainty With Public And Private Financing". Transport Policy, vol. 10, n. 2, pg. 141-156. 
Vancza, J., Egri, P. e Karnok, D. (2010) Planning in concert: a logistics platform for production networks. International Journal of Computer Integrated Manufacturing. Vol. 23, n. 4, p. 297307.

Wagner, T. (2010) Regional traffic impacts of logistics-related land use. Transport Policy, vol. 17, n. 4, pp. $224-229$.

Williamson, O. E. (1991) "Comparative Economic Organization: The Analysis of Discrete Structural Alternatives". Administrative Science Quarterly, vol. 36, n. 2, pp. 269-296.

Williamson, O. E. (1999) Strategy research: governance and competence perspectives. Strategic Management Journal, vol. 20, n. 12, pp. 1087-1108. 